Transactions of the Royal Society of Tropical Medicine and Hygiene (2006) xxx, xxx-xxx

available at www.sciencedirect.com

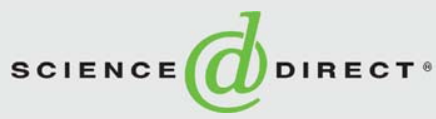

journal homepage: www.elsevierhealth.com/journals/trst

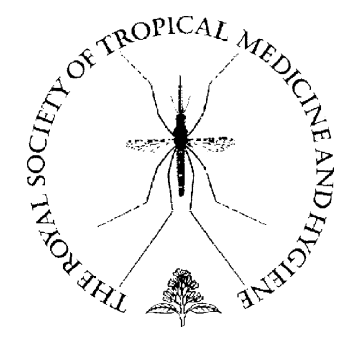

SHORT COMMUNICATION

\title{
Natural transmission of Plasmodium knowlesi to humans by Anopheles latens in Sarawak, Malaysia
}

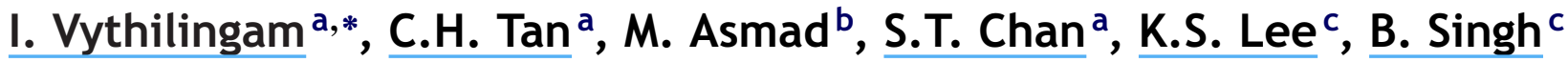

\author{
a Infectious Diseases Research Centre, Institute for Medical Research, Jalan Pahang, 50588 Kuala Lumpur, Malaysia \\ b State Health Department, Kuching, Sarawak, Malaysia \\ c Faculty of Medicine and Health Sciences, Universiti Malaysia Sarawak, Kuching, Sarawak, Malaysia
}

Received 30 November 2005; received in revised form 13 February 2006; accepted 13 February 2006

\section{KEYWORDS \\ Malaria; \\ Plasmodium \\ knowlesi; \\ Anopheles latens; \\ Malaysia}

\begin{abstract}
Summary Four species of malaria parasites are known to infect humans. A fifth species, Plasmodium knowlesi, has been reported to infect humans in Malaysian Borneo. Here we report for the first time the incrimination of Anopheles latens as the vector of $P$. knowlesi among humans and monkeys in Sarawak, Malaysia.

(c) 2006 Royal Society of Tropical Medicine and Hygiene. Published by Elsevier Ltd. All rights reserved.
\end{abstract}

\section{Introduction}

Malaria remains a public health problem in many countries in Southeast Asia. Besides the four species of Plasmodium that commonly cause malaria in humans, simian malarias such as Plasmodium inui, $P$. cynomolgi and $P$. knowlesi can also cause malaria in humans (Garnham, 1966). These simian malaria infections were acquired by humans through blood passage or in laboratory settings through mosquito bites (Garnham, 1966). Naturally-acquired cases of $P$. knowlesi in humans were thought to be extremely rare, as previously there had been only two reports of such cases, both in peninsular Malaysia (Chin et al., 1965; Yap et al., 1971). However, a large number of human $P$. knowlesi cases have recently been reported in Kapit, Sarawak, Malaysian Borneo by Singh et al. (2004). Cases are also being reported from peninsular

\footnotetext{
* Corresponding author. Tel.: +60 34040 2437; fax: +60 32694872. E-mail address: indra@imr.gov.my (I. Vythilingam).
}

Malaysia (I. Vythilingam et al., unpublished data). Plasmodium knowlesi has also been detected in a patient who had spent time in a forested area on the Thai-Myanmar border (Jongwutiwes et al., 2004). Thus, it is essential to identify the mosquito vectors responsible to determine the dynamics of $P$. knowlesi transmission to humans. Studies were therefore initiated to determine the vectors of monkey malaria in different areas in Kapit, Sarawak.

\section{Methods}

\subsection{Mosquito collection}

Mosquitoes attracted to humans and monkeys were collected and dissected. When sporozoites or oocysts were encountered, they were preserved in absolute ethanol. DNA was extracted using the Qiagen DNeasy tissue ${ }^{\circledR}$ kit (Qiagen, Hilden, Germany) and samples were analysed by means of a nested PCR-based assay (Singh et al., 2004). 